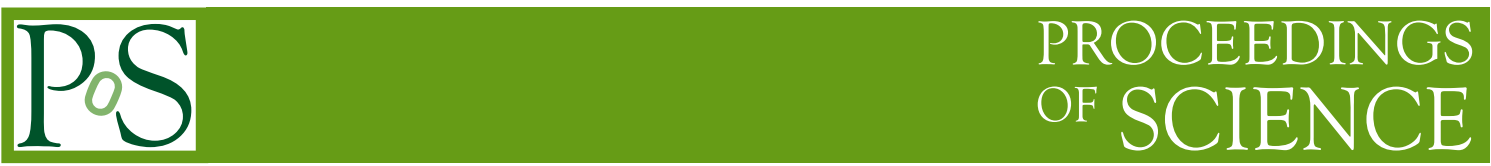

\title{
Ageing tests of the Hybrid Modules for the ALICE ITS Upgrade
}

\author{
Nicolò Valle*, on behalf of the ALICE collaboration \\ University and INFN Pavia, Italy \\ E-mail: nicolo.valle@cern.ch
}

The ALICE experiment foresees a comprehensive upgrade during the ongoing long LHC shutdown. A key element is the replacement of the Inner Tracking System (ITS) with a newly constructed silicon based detector allowing a significant improvement of the tracking and vertexing capabilities. Several institutions were involved in the assembly of the modules (HICs, Hybrid Integrated Circuits) of the new ITS. Dedicated hardware and software were developed in order to qualify the modules at different stages after the assembly. Some HICs underwent more invasive tests designed to check their mechanical strength. These include ageing tests: by keeping the modules in a temperature and humidity controlled environment, their ageing in the ALICE cavern can be simulated. The pixel response and the quality of the HICs before and after different ageing periods were measured and compared, drawing conclusions on their stability over time during the data taking period. The HIC qualification procedure will be presented and particular emphasis will be given to the ageing tests.

The 28th International Workshop on Vertex Detectors - Vertex2019

13-18 October, 2019

Lopud, Croatia

${ }^{*}$ Speaker. 


\section{Upgrade of the ALICE Inner Tracking System}

The ALICE experiment has been designed to study the properties of the hot nuclear matter (quark-gluon plasma, QGP) created in heavy-ion collisions at LHC. Several experimental handles are available to extract information on the QGP and ALICE is playing a significant role in their measurement. During the Run3 and Run4 data-taking periods ALICE will focus on the measurement of heavy-flavor hadrons, quarkonia and low mass dileptons over a wide range of transverse momenta. To achieve these physics goals the detector is undergoing a comprehensive upgrade which will allow the experiment to improve its readout rate and tracking capabilities [1]. A cornerstone of the upgrade is the replacement of the Inner Tracking System (ITS) with a new detector fully based on innovative pixel sensors and fabricated on substrates with a high-resistivity epitaxial layer [2]. Each sensor (the ALPIDE chip, ALICE PIxel DEtector) is produced with TowerJazz technology ${ }^{1}$ based on $180 \mathrm{~nm}$ CMOS imaging process and contains half a million pixel sensors [3]. Pixels are less than $100 \mu \mathrm{m}$ thick $(50 \mu \mathrm{m}$ for those installed in the innermost part of the detector) and contain both a sensing diode and the readout electronics. The detector has been optimised to have a very low power consumption (less than $\left.40 \mathrm{~mW} / \mathrm{cm}^{2}\right)$ and a high spatial resolution $(5 \mu \mathrm{m}$ over a large operational range, possible thanks to the $29 \mu \mathrm{m} \times 27 \mu \mathrm{m}$ pixel size). Other remarkable features are the low fake-hit rate (less than $10^{-10}$ pixel/event), the large detection efficiency, and the low material budget $\left(0.35 \% X_{0}\right.$ for the innermost layers).

\section{The Hybrid Integrated Circuits}

The pixel chips cover a total active area of $\sim 10 \mathrm{~m}^{2}$ and are arranged in seven concentric cylindrical layers. The first three innermost layers are part of the Inner Barrel (IB) and are located between 2.2 and $4 \mathrm{~cm}$ in radial direction. The other four layers compose the Outer Barrel (OB), with the outermost one having a radius of $40 \mathrm{~cm}$. The length in the $z$ direction ranges from $27 \mathrm{~cm}$ (IB) to $148 \mathrm{~cm}$ (the two outermost layers). Each layer is azimuthally segmented in several staves. The smallest operable unit of each stave is called Hybrid Integrated Circuit (HIC) and consists of an array of pixel chips (9 or 14 chips for IB and OB HICs respectively). The picture of an OB HIC is visible in Fig. 1.

The IB HICs were produced at CERN while OB HICs were assembled in Bari (IT), Liverpool (UK), Pusan/Inha (IN), Strasbourg (FR) and Wuhan (CN). A common procedure and a partially automated assembly system are used to ensure an homogeneous quality among the different sites and during the whole production. As a first step the chips are aligned with a precision of maximum $5 \mu \mathrm{m}$. Then, by means of an adhesive mask, a precise pattern of glue droplets (epoxy resin) is dispensed on a polymide Flexible Printed Circuit (FPC) which is aligned and glued to the chips. An array of connection pads is present on the top surface of each chip. They are connected to the FPC for power supply and I/O with an ultrasonic wedge bonding using a $25 \mu \mathrm{m}$ aluminium wire. Three redundant connections are soldered on each pad (visible in Fig. 2). Aluminium and copper are used as FPC conductors for IB and OB modules respectively. The assembly of the FPCs and their quality inspection were performed in Trieste and Catania (IT).

\footnotetext{
${ }^{1}$ TowerJazz, https://www.jazzsemi.com/
} 


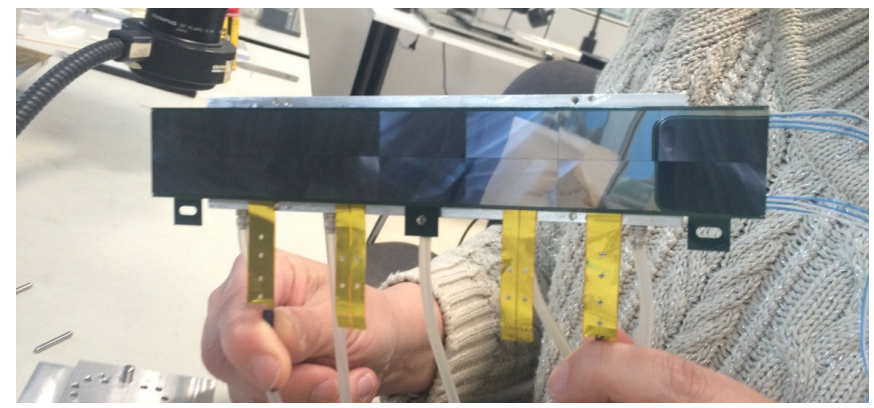

Figure 1: Sensor side of an Outer Barrel HIC.

Through custom electronics and interfaces several tests (referred to as Qualification Test) allow each module to be assessed after the assembly. A series of scans are performed and cuts to different parameters applied in order to classify the chips response. In the Digital Scan digital pulses are injected into the digital logic of the pixel cells. They are then read out and the number of pixels not responding is measured. In the Threshold Scan a charge is induced in the front-end circuit of the chips. By varying the induced charge, the charge threshold and noise are determined. A sub-sample of HICs undergo more destructive tests designed to measure their mechanical strength. During the Peel Test the FPC is ripped from the chips and the average breaking force along the HIC is measured. Another machine (Fig. 2) is dedicated to the Pull Test during which the wired bonds are pulled out for a well defined number of pads on the HIC. The distribution of the breaking force is measured, looking for low breaking forces $(<5 \mathrm{~g})$ and soldering damage.
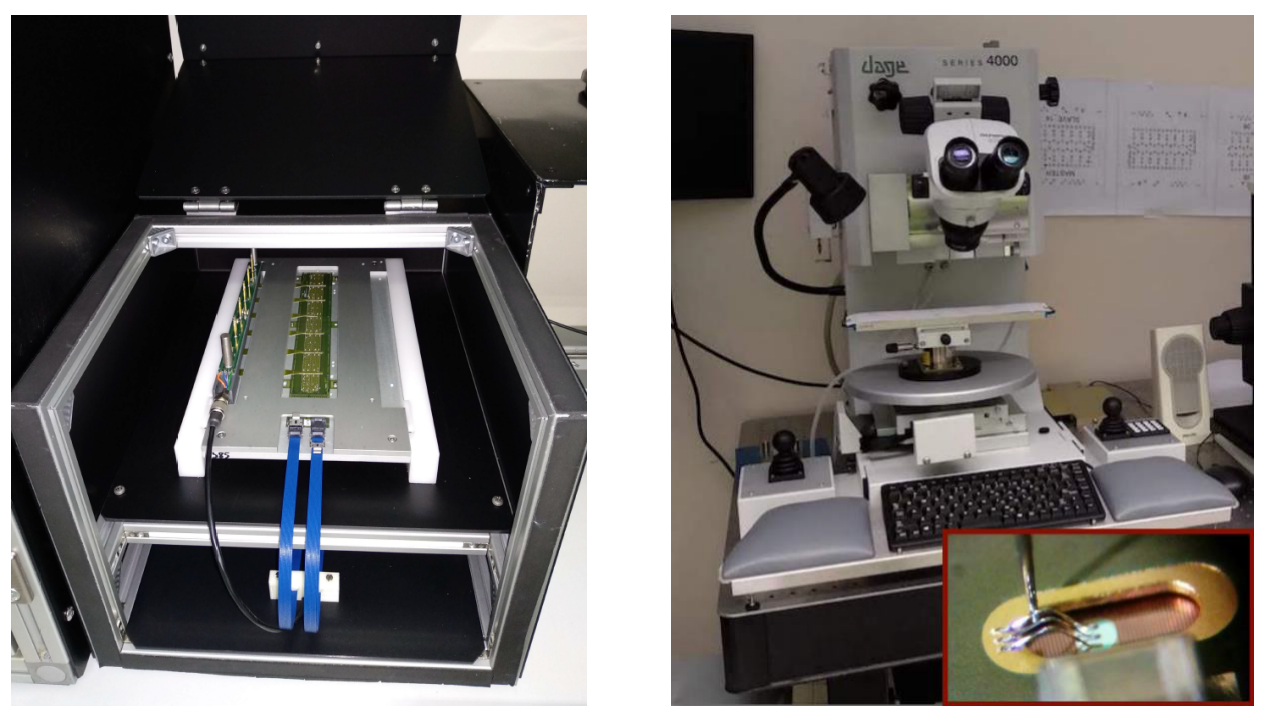

Figure 2: On the left: Outer Barrel HIC in the station used for the Qualification Test. On the right: the Pull Test station.

\section{Ageing tests}

Multiple series of ageing tests were performed in order to ensure the quality of the HICs and the staves for several years of operation. In this section we focus on the most recent results on $\mathrm{OB}$ 
HICs production. Previous results are described in [4]. A sub-sample of OB HICs were kept in a temperature and humidity controlled environment in order to simulate their ageing in the ALICE cavern. This allowed to check the endurance of the wire bonding, as well as the stability of the electrical connections and the pixel response.

Concerning the chip-FPC bonding, the Arrhenius relationship [5] can be used to describe the effects of the temperature and other factors, such as relative humidity, on the rate of oxidation reactions. According to this equation, the acceleration factor $(A F)$ induced by a temperature $T_{\text {stress }}$ is

$$
A F=\exp \left[\frac{E_{a}}{k_{B}}\left(\frac{1}{T}-\frac{1}{T_{\text {stress }}}\right)\right]
$$

where $E_{a}$ is the activation energy related to the reaction responsible of the soldering deterioration and $k_{B}$ the Boltzmann constant. $T=298 \mathrm{~K}$ is the operating temperature. According to reasonable estimates, with $E_{a}$ in the range $0.7-0.8 \mathrm{eV}$, a stress temperature $T_{\text {stress }}$ of $333 \mathrm{~K}$ causes an acceleration factor greater than 15 . The test was performed keeping the HICs in a climate chamber at $333 \mathrm{~K}$ and $15 \%$ relative humidity: in these conditions a period of 4 weeks is sufficient to simulate one year of real operation. The HICs underwent up to five equivalent years of ageing. After each period the HICs were tested, both mechanically (by means of the Pull Test) and electrically.

After each ageing cycle, 56 wire bonds per HIC were pulled out and the distributions of the breaking forces were compared to those obtained before the cycle. A sample of the mean and minimum values of the forces is shown in Fig. 3. Bonding damage can be quantified by the number of peel off and lift off events occurred during the Pull Test, meaning the number of detachments of the solder with or without removal of the metallization. As summarised in Fig. 4, the pad soldering seemed not to show thermal effect. The deterioration of the soldering on the copper FPC was more evident and found to be correlated with the decrease of the pull-force values. More in-depth studies didn't highlight a correlation between the low measured forces and the pad involved in the test, confirming that the redundant connections keep the system reliable.

On the other hand, the results of the qualification tests were used to monitor the HIC performances over time. The comparison of Qualification Test outputs before and after each cycle was done on both the entire HIC and the single chips. The number of bad pixels in Digital or Threshold Scana and the pixel noise are parameters of interest. The results didn't show any systematic effect due to the ageing: the number of working pixels as well as the noisy pixels and threshold distributions showed a good stability over time.

\section{Conclusions}

The ALICE Inner Tracking System is in the process of being replaced. Once installed, the new ITS will allow a significant improvement of the tracking and vertexing capabilities. Different series of ageing tests have been performed on the modules of the new detector which were kept under stress temperature and humidity conditions in order to ensure their stability during the data taking periods. The results of a recent analysis have been presented. The electrical tests demonstrated no difference in the performances of the pixels over time. On the other hand, a worsening of the soldering strength is expected and a decrease in the value of the wire-bonding breaking forces has been measured. However, the average value $(>10 \mathrm{~g})$ and the minimum value $(>5 \mathrm{~g})$ after 5 years, 

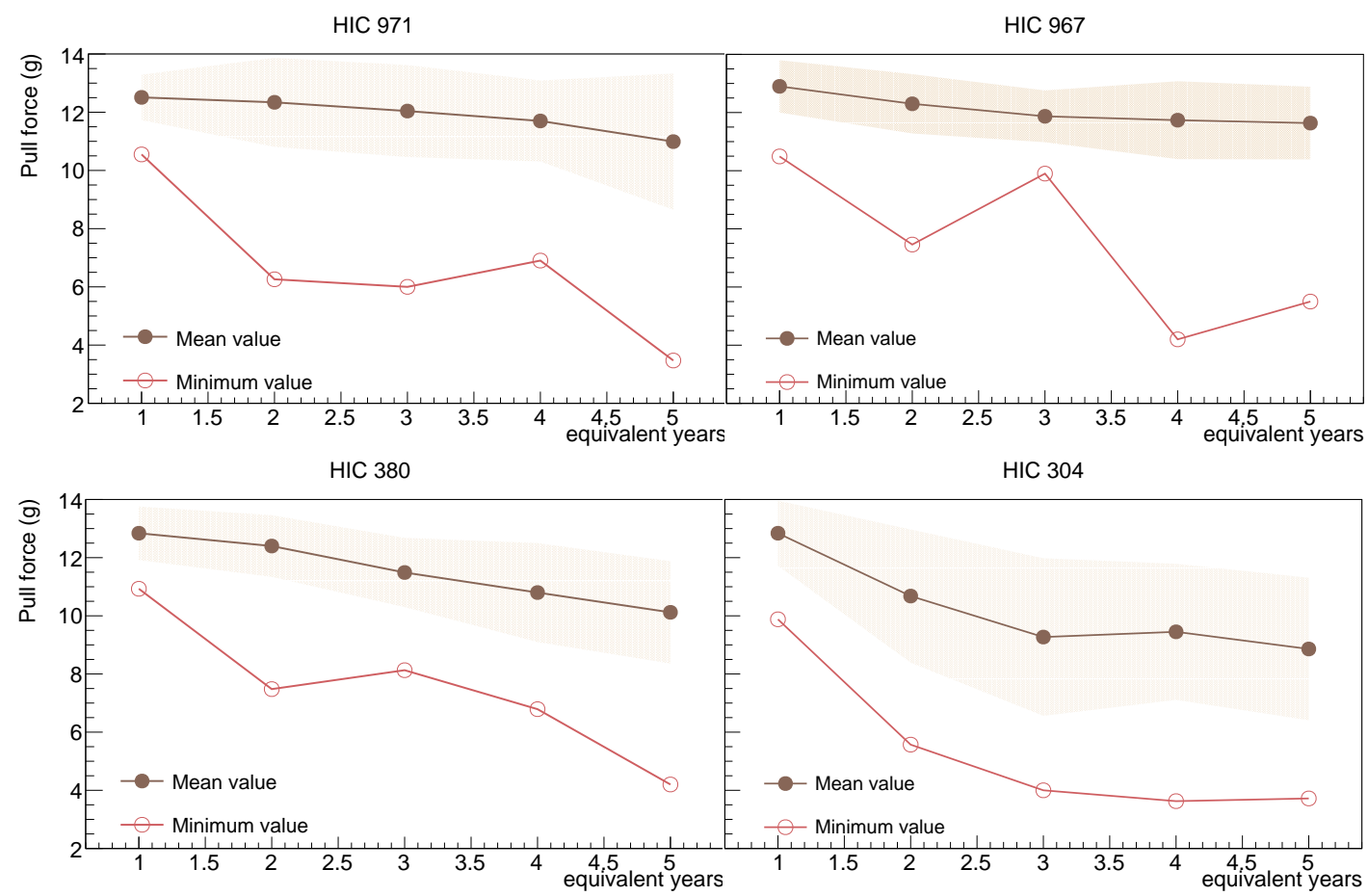

Figure 3: Distribution of the pull forces on four tested HICs as a function of time. The shadowed area is the standard deviation of the 56 measured values.

$$
40 \text { Number of peel off on CHIP }
$$
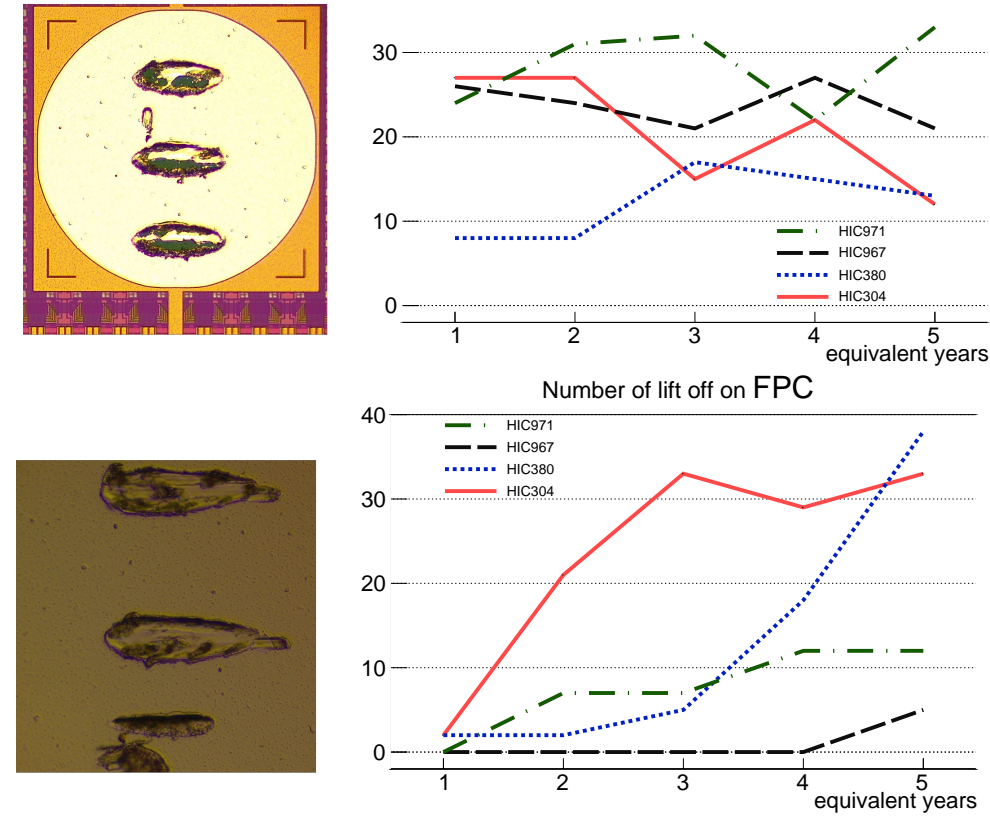

Figure 4: Peel off and lift off of the soldering on chip pad and FPC pad respectively. The plots report the evolution of the number of damages measured after each ageing period. Each line corresponds to a HIC. 
combined with the three-bond connections per pad, confirm the robustness of the system against ageing effects for the whole foreseen data taking period. Other studies based on thermal cycles are presented in [4]. In summary, the results of the ageing tests verified the assembly procedure and validated the use of the appropriate components.

\section{Acknowledgments}

We would like to thank the INFN Pavia Electronic Service for having provided support and part of the equipment used in the activities here described, in particular for the tests in the climate chamber.

\section{References}

[1] ALICE Collaboration, Letter of Intent for the Upgrade of the ALICE Experiment, J. Phys. G 41 (2014) 087001

[2] ALICE Collaboration, Technical Design Report for the Upgrade of the ALICE Inner Tracking System, J. Phys G 41 (2014) 087002

[3] G. Aglieri Rinella for he ALICE Collaboration, The ALPIDE pixel sensor chip for the upgrade of the ALICE Inner Tracking System, Nucl. Instrum. Meth. A845 (2017) 583-587

[4] D. Andreou, The upgrade of the ALICE Inner Tracking System, in proceedings of PIXEL 2018, JINST 14 (2019) 04

[5] L. A. Escobar, W. Q. Meeker, A Review of Accelerated Test Models, Statist. Sci. 21(2006) 4 